\title{
RELATIONSHIP BETWEEN BRAIN ACTIVITY AND REAL-ROAD DRIVING BEHAVIOR: A VECTOR-BASED WHOLE-BRAIN FUNCTIONAL NEAR-INFRARED SPECTROSCOPY STUDY
}

\author{
Yoshitomo Orino $^{1}$, Kouji Yamamoto ${ }^{2}$, Noriyuki Oka ${ }^{3}$, Hideki Takahashi ${ }^{4}$, Toshiyuki Sugimachi ${ }^{5}$, \\ Yoshihiro Suda ${ }^{5}$, Toshinori Kato ${ }^{3}$ \\ ${ }^{1}$ Traffic Control Center, Hachioji Branch, Central Nippon Expressway Co. Ltd. \\ ${ }^{2}$ Department of Environment/Engineering, Tokyo Branch, Central Nippon Expressway Co. Ltd. \\ ${ }^{3}$ Department of Brain Environmental Research, KatoBrain Co. Ltd. \\ ${ }^{4}$ Central Nippon Highway Engineering Nagoya Co., Ltd. \\ ${ }^{5}$ Institute of Industrial Science, The University of Tokyo, Japan \\ E-mail: h.takahashi.a@c-nexco-hen.jp
}

\begin{abstract}
Summary: Automobile driving requires multiple brain functions. However, the brain regions related to driving behavior are unknown. Therefore, we measured activity of the frontal, parietal and occipital lobes during driving using functional near-infrared spectroscopy (fNIRS). Cortical activation patterns were examined in relation to driving behaviors, such as steering motion, accelerator pedal motion, and speed control. Six healthy adults participated in the experiment. Cerebral oxygen exchange (COE) was calculated based on the oxyhemoglobin and deoxyhemoglobin concentrations measured by fNIRS. The COE and driving behavior data were collected every $1 \mathrm{~m}$ and averaged for all subjects. Functional NIRS data for all 98 channels were extracted using principal component analysis. Similarity between extracted components and driving behaviors were confirmed by $\mid$ cosine similarity $\mid>0.3$. Among the factors with confirmed similarity, we identified brain regions with high principal component loading ( $\mathrm{PCL} \mid>0.4)$. Among the $16 \mathrm{COE}$ factors extracted, COE factor 1 and factor 5 exhibited similarity with steering motion (cosine similarity: factor $1,-0.538$; factor 5 , 0.551). The PCLs of COE factor 1 and factor 5 were high in the frontal lobe (Brodmann areas [BAs] 9, 8, and 4/3) (PCL $>0.8)$. COE factor 6 exhibited a similarity with accelerator pedal motion (cosine similarity: 0.369), and the PCL of COE factor 6 was highest in the parietal lobe $(\mathrm{BA7})(\mathrm{PCL}=-0.62)$. Speed control did not exhibit similarity with any COE factor. These findings will contribute to the selection of brain measurement areas when fNIRS is used for vehicle driving assessment.
\end{abstract}

\section{OBJECTIVES}

Regardless of the road environment, traffic congestion and traffic accidents ultimately depend on human factors. Therefore, establishment of methods to assess human factors while driving on real roads, although challenging, may lead to improved traffic safety measures. However, previous survey methods of human factors were based on subjective data such as introspective questionnaire reports and traffic data. Thus, no study has employed methods that are free of survey bias to evaluate drivers' reactions while on the road. Evaluation of physiological indicators is considered an effective strategy to circumvent these problems. Therefore, we have been working on ways to measure brain activity related to cognition, judgment, and movement during real automobile driving. 
There are three technical requirements for functional brain measurements while driving: realtime measurement, minimal signal noise in the vehicle environment, and non-invasiveness. Brain functional near-infrared spectroscopy (fNIRS), in which the skull surface is irradiated by nearinfrared light and scattered reflected light is detected as a measure of cortical blood oxygenation, fulfills all three requirements. We previously reported that actual vehicle operation differentially activates frontal and parietal cortices as measured by fNIRS (e.g., Yoshino, 2013). However, most driving research using fNIRS has focused only on activity in the prefrontal cortex because of the limited number of channels available in commercial fNIRS systems (e.g., Harada et al, 2007; Tsunashima, 2009; Foy et al, 2016), while the regions most relevant to driving remain unclear. Thus, thorough investigation of the human factors involved in driving requires fNIRS analysis over a larger area of the brain.

In this study, brain regions contributing to steering and accelerator motion were detected for the first time using statistical analysis of a wide range of measurements taken from areas including the frontal, parietal, and occipital lobes.

\section{METHODS}

\section{Participants}

The participants included six right-handed healthy adults ( 2 male and 4 female; average age: $35.3 \pm 3.6$ years old) that had held driver's licenses for an average of $14.2 \pm 5.0$ years. Participants had no history of central nervous system disease or mental illness. All procedures in this study were approved by the ethics committee of the University of Tokyo. Research objectives were explained both in writing and orally, and all participants provided written informed consent for participation and reporting of the results.

\section{Experiment}

Experimental field and car. The experiment was performed in the outer peripheral path of the Shirosato Test Center, Japan Automobile Research Institute (General Incorporated Foundation, Ibaraki Prefecture, Japan). The experiment course is a two-lane road with 3.75-m lane widths. Of the total 5677-m length, the interval from $3928 \mathrm{~m}$ to $5673 \mathrm{~m}$, where the road takes several turns (Figure 1), was used for evaluation.

The test vehicle was a regular passenger car (Estima hybrid, TOYOTA, Japan) equipped with an fNIRS system to measure brain activity. The vehicle position was measured by the GPS signal, while the speed, steering, and stroke of the accelerator and brake pedal were monitored by controller area network information. 


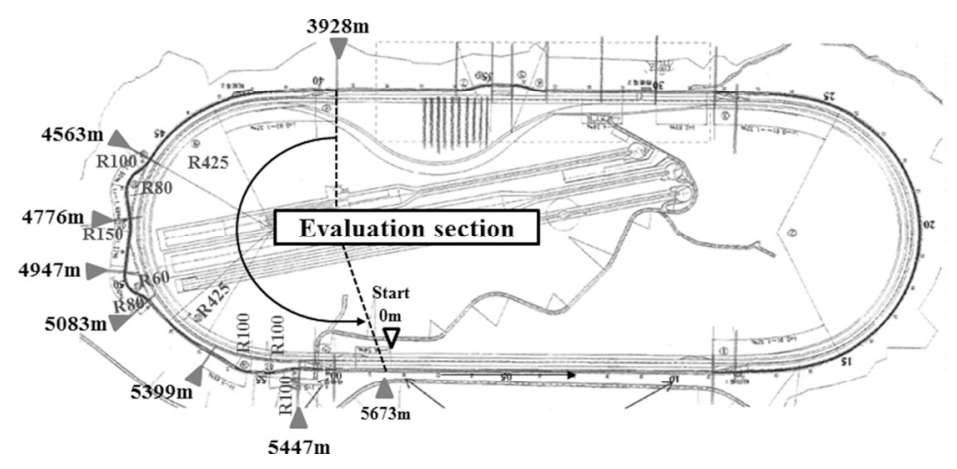

Figure 1. Experiment field

fNIRS device and real-time monitoring of brain activity during driving. Multi-channel fNIRS (FOIRE-3000, Shimadzu Corporation, Japan) was used for brain activity measurement. fNIRS can monitor oxyhemoglobin $(\mathrm{OxyHb})$ and deoxyhemoglobin (DeoxyHb) concentrations by irradiation of three different wavelengths of near-infrared light $(780,805$, and $830 \mathrm{~nm})$ from the scalp (e.g., Kato, 1993). The measurement was performed with a total of 98 channels using 16 irradiation and 16 detection probes. To measure 98 channels, we used two probe folders (Figure $2 \mathrm{a}, \mathrm{b}$ ). The measurement sites of the first probe folder configured with 46 channels (Probe V) were over both sides of the prefrontal cortex [Brodmann area (BA) 9 and 45], frontal eye fields (BA8), supplementary motor area (BA6), visual cortex (BA19, 18, and 17), and cerebellum (Figure 2a). The measurement sites of the second probe folder configured with 52 channels (Probe $\mathrm{M}$ ) were supplementary motor area (BA6), primary sensory and motor area (BA4/3), and parietal association area (BA7, 40).
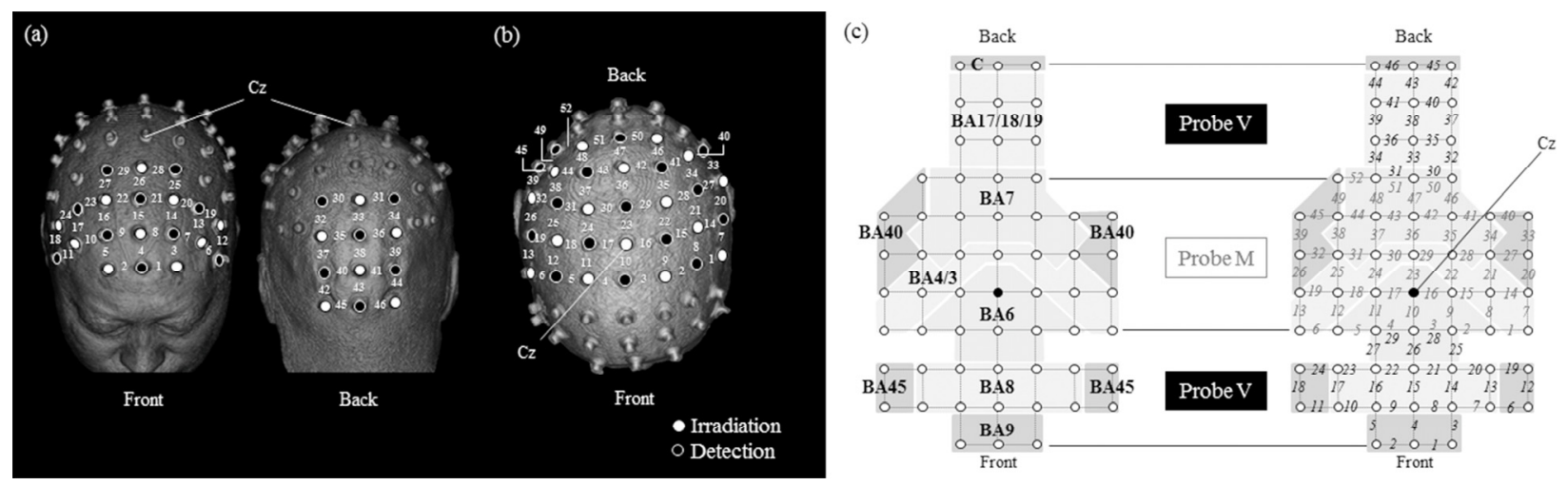

Figure 2. fNIRS measurements

(a) Measurement sites of Probe V. (b) Measurement sites of Probe M. (c) Correspondence between the measurement channels of Probe $\mathrm{V}$ and $\mathrm{M}$ with Brodmann areas.

Each probe folder was mounted based on the $\mathrm{Cz}$ landmark of the international 10-20 system (Figure 2a, b). The position of each measurement channel was confirmed by 3D T2-weighted MRI images acquired using a 3.0-T Achieva 3.0 Quasar Dual system (Philips). Participants wore attachments with registration markers during MRI imaging. From the position of the registration markers, the probe installation position and measurement sites were identified. The BAs shown in Figure $2 \mathrm{c}$ are those with the highest matching rates to MRI images from all participants. 
Experiment procedure. Participants drove the experimental course counter-clockwise three times with Probe V and three times with Probe M. Based on the Japanese Road Traffic Act, participants drove in the left lane. The estimated travel speed was set to $80 \mathrm{~km} / \mathrm{h}$.

\section{Data analysis}

Analysis of brain activity. Changes in cerebral oxygen exchange $(\triangle \mathrm{COE})$ were calculated as a metric of oxygenation changes in cerebral capillaries (and associated neural activity) based on fNIRS measurements of changes in $\mathrm{OxyHb}$ concentration $(\triangle \mathrm{OxyHb})$ and DeoxyHb concentration $(\triangle \mathrm{DeoxyHb})$. Increased $\mathrm{COE}$ indicates that oxygenation in blood vessels is low, whereas decreased COE indicates higher oxygenation in blood vessels. $\triangle \mathrm{COE}$ is a more accurate physiological indicator of localized brain activity than the conventionally used $\triangle \mathrm{OxyHb}$ or cerebral blood volume changes (e.g., Kato, 2004; Yoshino, 2013; Oka, 2015). $\Delta$ COE was calculated from equation (1) (e.g., Kato, 2004).

$$
\triangle C O E=\frac{\Delta D e o x y H b-\Delta O x y H b}{\sqrt{2}}
$$

To evaluate and analyze associations between participant behavior and brain activity, both data sets were normalized to the travel distance (e.g., Kato, 2012). Therefore, the data were linearly interpolated using GPS coordinates with respect to the locus on the road for every $1 \mathrm{~m}$. The travel start point was set to zero ( 0 -m point) for the COE value of average land-series data. The purpose of this analysis was to identify the brain regions exhibiting activation (changes in COE) associated with three driving behaviors: steering, accelerator operation, and speed changes. The components were identified by principal component analysis (PCA) using average COE landseries data normalized every $1 \mathrm{~m}$ from all 98 channels of Probe V and M. Using PCA, we extracted several components that summarized 98-channel data. The similarity between extracted components and the three driving behaviors are expressed by cosine similarity. The presence of a similarity for a given component was defined by a cosine similarity of \pm 0.3 or higher (absolute value of 0.3 ).

For components with an absolute value of 0.3 or higher cosine similarity to driving behavior, mapping analysis was performed based on the value of principal component loadings (PCLs). The brain regions related to each principal component were identified based on the absolute value of the PCL of 0.4 or higher (e.g., Murase et al, 2007). Analysis was performed using SPSS statistics ver. 23 (IBM, Japan). In total, 16 trials from Probe V (4 subjects $\times 3$ trials, 2 subjects $\times 2$ trials) and 15 trials from Probe M (5 subjects $\times 3$ trials) were acquired without missing data points.

\section{RESULTS}

\section{Degree of similarity between driving behavior and factor of brain activity ( $\triangle \mathrm{COE}$ )}

We extracted $16 \mathrm{COE}$ factors with eigenvalues of 1.0 or higher by PCA. Among these $16 \mathrm{COE}$ factors, we detected a similarity of factor 1 and factor 5 with steering behavior (Cosine similarity: factor 1, -0.538; factor 5, 0.551) (Figure 3a, 4b). We also detected a similarity between COE factor 6 and accelerator operation (Cosine similarity: 0.370) (Figure 3c). We did not detect any similarity to other factors. No factor was similar to speed. 

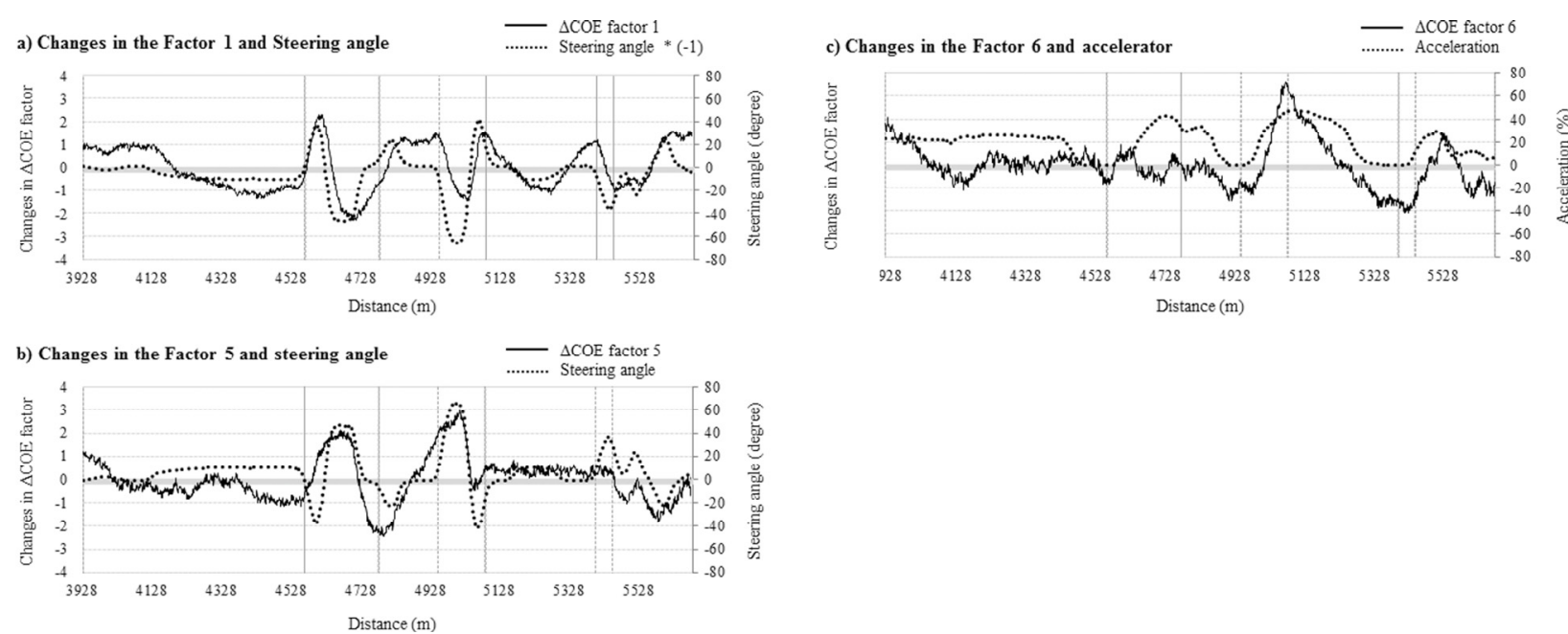

Figure 3. Land-series waveform of driving behavior and fNIRS data

(a) Waveform of factor 1 and steering angle. For steering angle, a positive value indicates steering to the right, and a negative value indicates steering to the left. (b) Waveform of factor 5 and steering angle. In this case, a positive value indicates steering to the left, and a negative value indicates steering to the right. (c) Land-series waveform and accelerator operation.

\section{Brain regions contributing to steering}

Figure 4 shows mapping images of PCL from all measurement channels against factors 1 and 5, for which we detected a similarity to steering behavior. Figure 4a shows PCL against COE factor 1, and Figure $4 \mathrm{~b}$ shows that against $\mathrm{COE}$ factor 5 . The brain regions with PCLs of absolute value 0.4 or higher for COE factor 1 were bilateral BA9, BA8, BA6, BA4/3, and BA7; right BA40, BA17, and BA19; and left cerebellum. The brain regions with PCLs of absolute value 0.4 or higher for COE factor 5 were right BA8, left BA6, right BA4/3, bilateral BA7, left BA40, and left BA19/18/17. Among these, PCLs of absolute value 0.8 or higher were detected in bilateral BA9 (left: channel 3; right: channel 5), bilateral BA8 (left: channel 7; right: channel 10), and right BA4/3 (channel 30). These results suggest that steering behavior is linked to activation in a broad region of the cortex, including the frontal, parietal, and occipital lobes. Among these regions, the contribution of bilateral frontal lobe (BA8 and BA9) and right BA4/3 is high. The factor contribution ratio and cumulative contribution ratio for factor 1 were $17.75 \%$ each. For factor 5 , the contribution ratio was $7.42 \%$ and the cumulative contribution ratio was $56.51 \%$.

\section{Brain regions contributing to accelerator operation}

For factor 6, which showed a similarity to accelerator operation, the brain regions showing PCLs of absolute value 0.4 or higher were found in the frontal lobe (BA8, BA6, BA4/3), parietal lobe (BA7, BA40), and occipital lobe (BA17) (Figure 4c). Among these, right BA7 (channel 37, channel 48) showed the highest PCL (0.62), suggesting a higher contribution of right BA7 to accelerator behavior. For factor 6 , the contribution ratio was $6.07 \%$ and the cumulative contribution ratio was $62.58 \%$. 


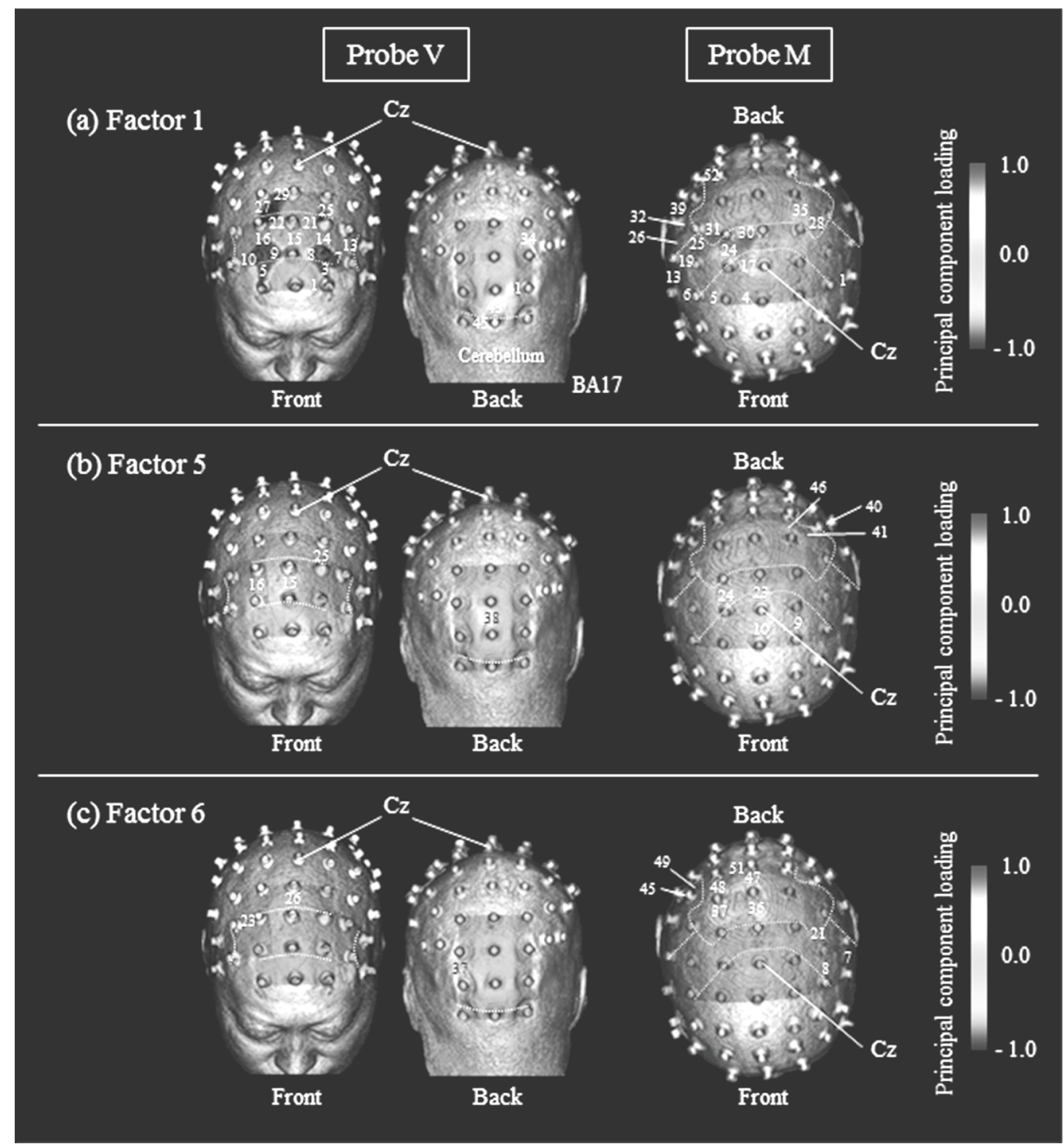

Figure 4. PCL mapping of factor 1, factor 5, and factor 6

PCL map of each channel against factor 1 (a), factor 5 (b), and factor $6(\mathrm{c})$. The numbers in the figure are the channel IDs for Probe V and Probe M.

\section{CONCLUSION}

fNIRS is the only brain measurement method that can detect brain activity that reflects driving behavior in real time during actual vehicle operation. Through the wide range measurements of the cerebral cortex by 98 channels, the present study was the first to statistically find that steering is mainly related to frontal lobe activity and that accelerator pedal motion is mostly associated with parietal lobe activity. The measurement range of fNIRS is limited to the number of channels of the apparatus. Our findings are useful for selecting the measurement sites of fNIRS for evaluating road safety measures. We believe that the evaluation of a driver's real-time reaction by fNIRS will be a powerful evaluation method not only for evaluating the driver but also for creating new knowledge to build an optimal road environment for the driver. 


\section{ACKNOWLEDGEMENTS}

This study presents partial results from the Brain Science Working Group, Traffic Information Service Study Group, and Central Japan Tokyo Office of NEXCO.

\section{REFERENCES}

Foy, H.J., Runham, P., Chapman, P. (2016). Prefrontal cortex activation and young driver behaviour: a fNIRS study. PLoS ONE, 11(5), e0156512.

Harada, H., Nashihara, H., Morozumi, K., Ota, H., Hatakeyama, E. (2007). A comparison of cerebral activity in the prefrontal region between young adults and the elderly while driving. Journal of Physiological Anthoropology, 26(3), 409-414.

Kato, T. Apparatus for evaluating biological function. United States Patent : US7065392. 2006. http://www.wipo.int/patentscope/search/en/WO2003068070. Accessed July 28, 2014.

Kato, T. Biofunction measurement device, biofunction measurement method and program. .Japanese Patent 2012289153. http://astamuse.com/ja/published/JP/No/2014128495. Accessed July 11, 2016.

Kato, T. Biofunctuon diagnosis device, biofunction diagnosis method, bioprobe, bioprobe wearing tool, bioprobe support tool, and bioprobe wearing assisting tool. European Patent: EP1818016. WO/2006/009178. 2007. http://www.wipo.int/patentscope/search/en/WO2006009178. Accessed July 28, 2014.

Land, M.F., Lee, D.N. (1994). Where we look when we steer. Nature, 169(6483), 742-744.

Liu, T., Pelowski, M., Pang, C., Zhou, Y., Cai, J. (2016). Near-infrared spectroscopy as a tool for driving research. Ergonomics, 59(3), 368-379.

Mars, F., Navarro, J. (2012). Where we look when we drive with or without active steering wheel control. PLOS ONE 7(8): e43858.

Phinney, R.E., Siegei, M. (2000). Speed selectivity for optic flow in area 7a of the behaving macaque. Cerebral cortex, 10, 413-421.

Pierrot-Deseilligny, C., Milea, D., Muri, R.M. (2004). Eye movement control by the cerebral cortex. Current Opinion in Neurology, 17(1), 17-25.

Sarter, M., Gicens, B., Bruno, J.P. (2001). The cognitive neuroscience of sustained attention: where top-down meets bottom-up. Brain Research Reviews, 35(2), 146-160.

Tsunashima, H., Yanagisawa, K. (2009). Measurement of brain function of car driver using functional near-infrares spectroscopy (fNIRS). Computational Intelligence and Neuroscience, 2009, 12.

Yoshino, K., Oka, N., Yamamoto, K., Takahashi, H., Kato, T. (2013). Functional brain imaging using near-infrared spectroscopy during actual driving on an express-way. Frontiers in Human Neuroscience, 7, 882.

Murase, Y., Takada, H., Hirose, T. (eds) Multivariate Analysis by SPSS. Ohmsha Ltd. Publication, 2007, 230. 